\title{
Non-Genetic Generation of Antibody Conjugates Based on Chemoenzymatic Tyrosine Click Chemistry
}

\author{
Jorick J. Bruins, Johannes A. M. Damen, Marloes A. Wijdeven, Lianne P. W. M. Lelieveldt, \\ Floris L. van Delft, and Bauke Albada*
}

Cite This: https://doi.org/10.1021/acs.bioconjchem.1c00351

Q Read Online

ACCESS | Lلll Metrics \& More | 回 Article Recommendations

ABSTRACT: The availability of tools to generate homogeneous and stable antibody conjugates without recombinant DNA technology is a valuable asset in fields spanning from in vitro diagnostics to in vivo imaging and therapeutics. We present here a general approach for the conjugation to human IgG1 antibodies, by employing a straightforward two-stage protocol based on antibody deglycosylation followed by tyrosinase-mediated orthoquinone strain-promoted click chemistry. The technology is

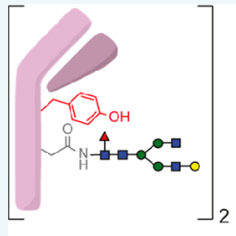

Native IgG1

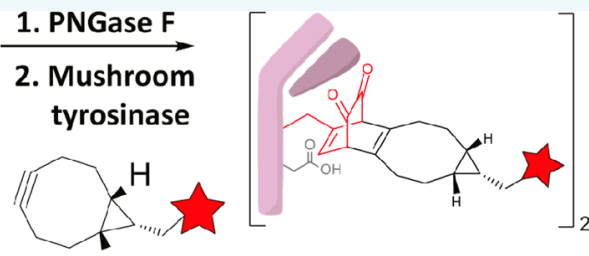

$\mathrm{H}$

Antibody-Drug Conjugate cytotoxic payloads MMAE or PBD dimer, and their in vitro evaluation.

\section{INTRODUCTION}

The attachment of functional modalities to monoclonal antibodies (mAbs) is key in the fields of diagnostics, ${ }^{1}$ imaging, ${ }^{2-4}$ and therapeutics. ${ }^{4,5}$ To achieve precisely controlled conjugation, usually a recombinant antibody is subjected to a chemoselective reaction with a functional payload, e.g., an affinity tag, ${ }^{6}$ a fluorophore, ${ }^{7}$ or a radionuclide. ${ }^{4}$ Alternatively, it is attached to a macromolecular structure, e.g., a vesicle, a nanoparticle, a microchip, or a solid surface. ${ }^{8}$ For example, key recognition events in ELISA are based on the highly specific capture of a specific antigen, a principle that is also applied in diagnostic sensors, such as pregnancy or COVID-19 test kits.' Functional antibodies are also heavily employed in clinical settings, such as molecular imaging (labeling with radionuclides), ${ }^{4}$ intraoperative guidance tools (labeling with fluorophores), ${ }^{10}$ and therapy (labeling with $\alpha$ emitters or cytotoxic payloads). ${ }^{4}$ The latter application, i.e., targeted chemotherapy with antibody-drug conjugates (ADCs), can be traced back to the early pioneering work of Mathé in 1958, who demonstrated the prolonged survival of mice grafted with a leukemia cell upon administration of an antibody-drug cocktail in which the conjugates were obtained by treatment of the antibody with diazotized methotrexate. ${ }^{11}$ Nevertheless, the development of therapeutic ADCs has been a long and complex road that is characterized by dozens of clinical failures. This resulted in the temporary withdrawal of the single approved ADC (Mylotarg) due to lack of clinical benefit in $2010^{12}$ (it was reintroduced in 2017). The approval of Adcetris in 2011 for the treatment of non-Hodgkin lymphoma can be regarded as the key turning point in the appreciation of ADCs for targeted cancer therapy, ${ }^{13}$ resulting in a current total of $10 \mathrm{ADCs}$ that reached the market. That this is made possible by recent advances in chemistry is clear from the fact that five of the current nine were approved in the past 18 months alone. At the moment, more than 80 additional ADCs are in various stages of clinical evaluation. ${ }^{14}$

Inspection of the conjugation technologies employed in the approved $\mathrm{ADC}$ reveals that seven of these are prepared by a reduction-conjugation sequence that functionalizes interchain sulfides (Adcetris, Polivy, Padcev, Enhertu, Trodelvy, Blenrep, and Zynlonta) and three by stochastic lysine conjugation (Kadcyla, Besponsa, and Mylotarg). ${ }^{14,15}$ Based on the aspecific nature of conjugation, the vast majority of these ADCs are in fact highly heterogeneous mixtures, which are not only difficult to characterize but, more importantly, almost impossible to purify. As a result, a mixture is used in which each conjugate will display a distinct and potentially unfavorable pharmacokinetic profile. ${ }^{16}$ Therefore, significant focus has been directed to the generation of homogeneous $\mathrm{ADCs}$ by means of sitespecific modification of the parent antibody. In this, the seminal work by Genentech reported in 2008 on "THIOmab" ADCs obtained by engineering of an additional cysteine into the $\mathrm{mAb}$ protein sequence stands out as an early example (Figure 1). ${ }^{17}$ Similarly, groundbreaking work from the Schultz group at Scripps (and others) has applied genetic encoding of uniquely reactive non-natural amino acids (NNAA) for highly site-selective attachment of cytotoxic payloads (Figure 1). ${ }^{18,19}$

Received: July 14, 2021

Revised: August 30, 2021 

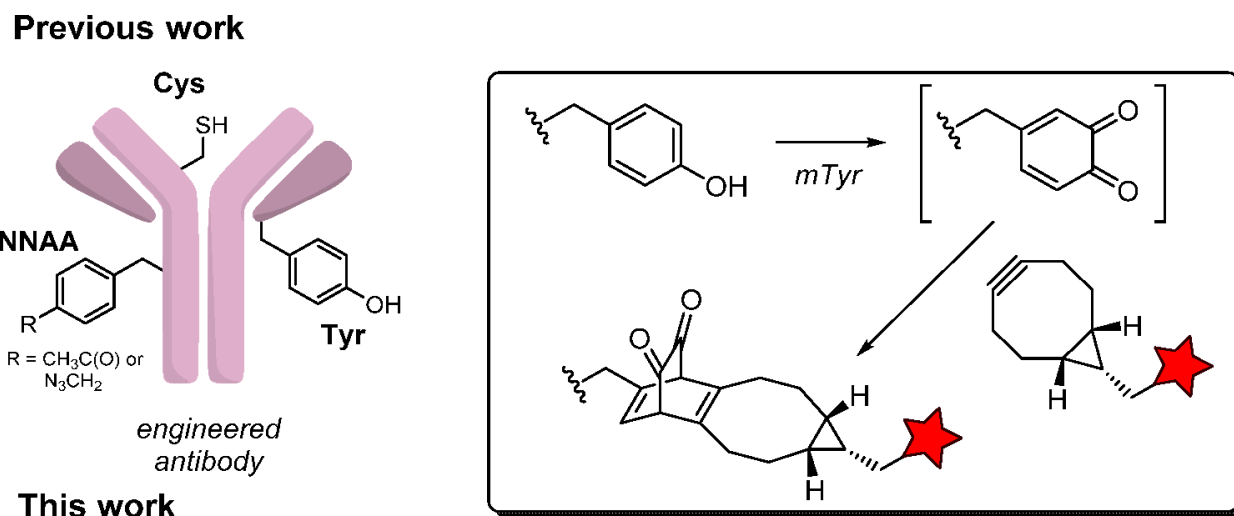

This work
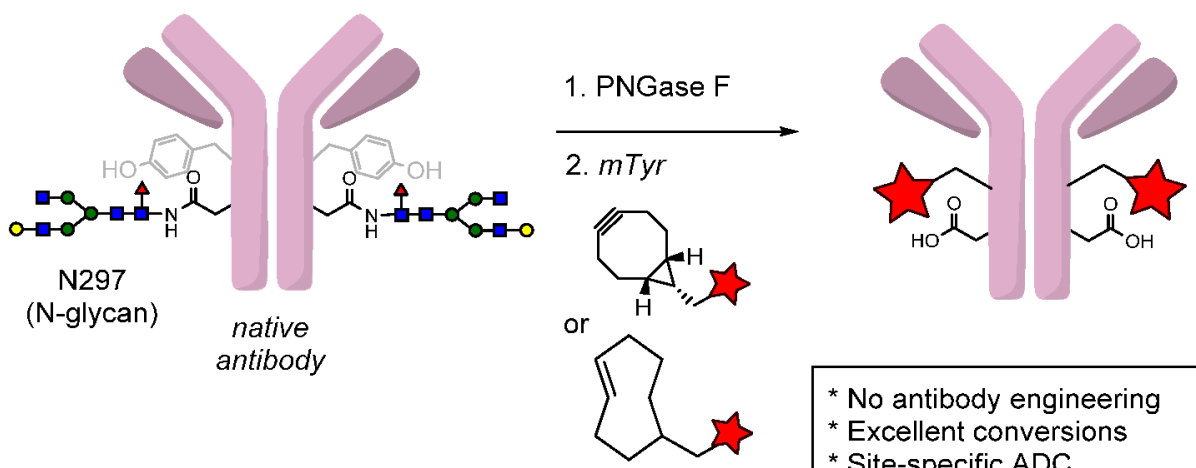

* No antibody engineering

* Excellent conversions

* Site-specific ADC

* Tailored stoichiometry

Figure 1. Generation of ADCs via SPOCQ chemistry on engineered antibodies (previous work) versus native antibodies (this work).

Today, almost $20 \%$ of all ADCs in clinical trials are prepared by one of these mentioned second-generation ADCs technologies, $^{20}$ i.e., cysteine engineering (at least 10) and genetic encoding of NNAA (at least 5). Furthermore, a multitude of other methods based on engineering of a specific amino acid or peptide tag has been developed for selective conjugation on a predetermined site, e.g., by enzymatic modification $^{21}$ and/or by a bio-orthogonal chemical reaction. $^{22,23}$

Efforts from our own group have revealed that a highly efficient and reliable process for site-specific and stable conjugation of chemical moieties to antibodies involves the engineering of a uniquely exposed tyrosine residue and its subsequent chemoenzymatic reaction with a label/drug (Figure 1). ${ }^{24,25}$ These efforts were based on the knowledge that native tyrosine residues, in general, are less exposed due to their hydrophobicity and tendency of $\pi-\pi$ stacking, resulting in them being poorly accessible for enzymatic modification. Thus, upon subjecting an antibody with an engineered exposed tyrosine-tag to mushroom tyrosinase (mTyr), the exposed tyrosine is converted into a highly reactive ortho-quinone that readily undergoes in situ cycloaddition with a strained alkyne, such as BCN, thereby forming a stable [2.2.2]-bicyclooctadiene conjugate (Figure 1, box), a process referred to as SPOCQ ( train-Promoted Oxidation-Controlled 1,2-Quinone cycloaddition). ${ }^{26-28}$ Notwithstanding the efficiency of the process and the stability of the resulting conjugates, we realized that the requirement of an engineered antibody could limit widespread application. Besides, conjugation of highly hydrophobic cytotoxic payloads at an exposed antibody site such as the $\mathrm{C}$ - or N-terminus can negatively impact the pharmacokinetic profile of the resulting $\mathrm{ADC},{ }^{29-31}$ and conjugation at a less-exposed site might be preferred.
In this paper, we report the efficient generation of antibody conjugates with SPOCQ technology upon enzymatic removal of the native N297 glycan (Figure 1, bottom). NMR-studies on antibodies with trimmed glycans already indicated substantial mobility of the $\mathrm{Y} 300$ residue, ${ }^{32}$ suggesting it might become sufficiently exposed to favor chemoenzymatic conversion. Indeed, we found that deglycosylation of human IgG1 antibodies sufficiently exposes a nearby $\mathrm{Tyr}$ residue and enables it to readily undergo tyrosinase-mediated oxidation to its ortho-quinone. In the presence of either a cyclooctyne (BCN) or trans-cyclooctene (TCO) it undergoes a SPOCQ cycloaddition, resulting in a conjugate in which the foreign moiety is added to the Fc domain. As such, it can be applied in the controlled conjugation of a range of functional payloads to nonengineered IgG1 antibodies. The Fc-fragment of a monoclonal antibody may be considered a privileged site for $\mathrm{ADC}$ generation, as it is remote from the antigen-binding site and is highly conserved across multiple antibodies. It is therefore not surprising that conjugation to the Fc-domain is applied in a multitude of clinical ADCs, including, for example, all ADCs based on S239C mutation or 239C insertion. ${ }^{14}$ Furthermore, the region around the N297 glycan has been reported as a particularly favorable site for the attachment of common cytotoxic payloads due to the natural hydrophobic cavity around the glycan. ${ }^{33}$ Various methods for modification of the antibody glycan have been reported, for example, early studies on periodate oxidation-oxime ligation of sialic acids ${ }^{34}$ or more recent enzymatic glycan remodeling, followed by strained alkyne-azide click chemistry conjugation of payloads. ${ }^{35}$ The latter approach is currently employed in three clinical ADC programs (ADCT-601, XMT-1592, and MRG004a). 
A

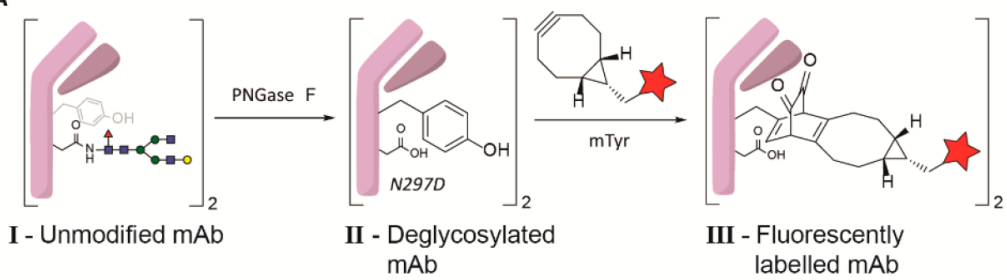
mAb labelled mAb

B
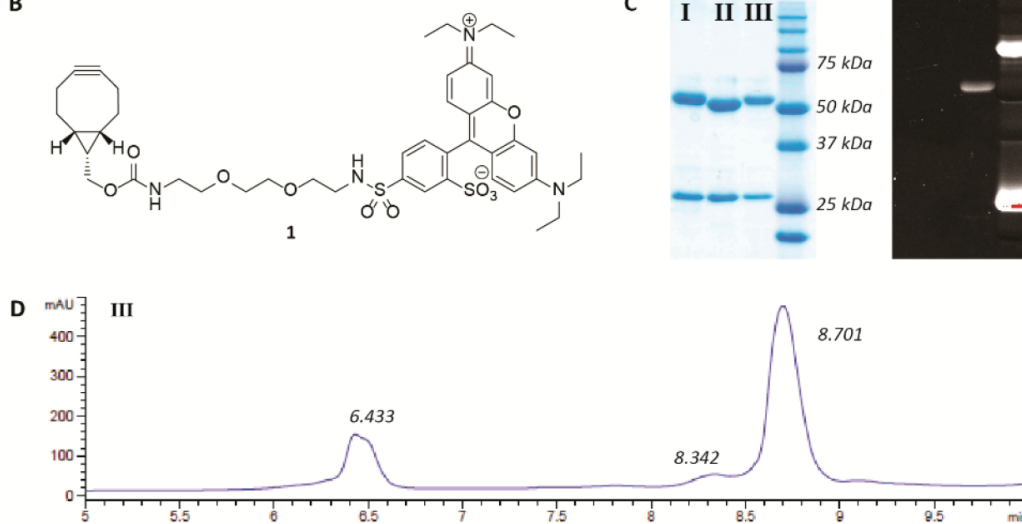

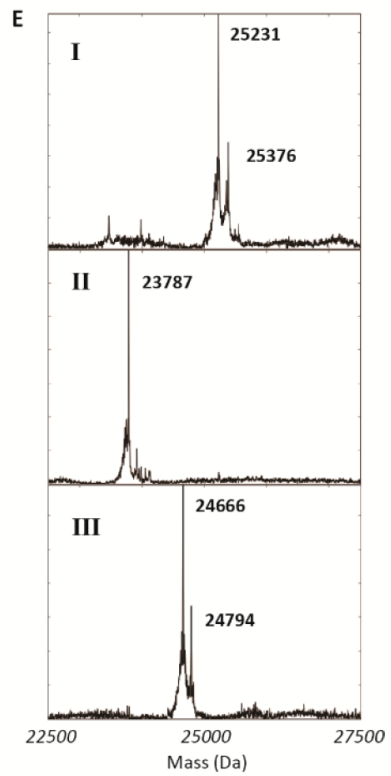

Figure 2. (A) Two-step modification of the monoclonal antibody trastuzumab, (B) structure of BCN-lissamine 1, (C) SDS-PAGE analysis of the two subsequent steps shown in panels A and B, (D) HPLC analysis of the final denatured product with the native light chain at $t_{\mathrm{R}}=6.4$ min and the lissamine-labeled heavy chain at $t_{\mathrm{R}}=8.7 \mathrm{~min}$, and $(\mathrm{E})$ deconvoluted spectra of the LC-MS analysis of the products obtained by the two steps of the modification of trastuzumab with BCN-lissamine 1.

\section{RESULTS AND DISCUSSION}

Inspired by the work of Schibli et al.-who reported that PNGase F digestion of the N297 glycan liberates glutamine Q295 in the Fc-domain, allowing for transglutaminasemediated ligation ${ }^{36}$-we reasoned that a neighboring tyrosine moiety could also be made available, as suggested by NMR studies. ${ }^{32}$ Therefore, antibody modification upon tyrosinasemediated oxidation followed by strain-promoted cycloaddition was proposed (Figure $2 \mathrm{~A}$ ). Although the constant $\mathrm{C}_{\mathrm{H}} 2$-domain of human IgG1 contains two conserved tyrosine residues (Y296 and Y300) that could potentially become sufficiently exposed upon PNGase F-mediated digestion, NMR studies indicate that mobility of Y300 exceeds that of Y296. ${ }^{32}$ Thus, to explore the feasibility of enzymatic glycan removal followed by tyrosinase-mediated cycloaddition, trastuzumab was treated with PNGase $\mathrm{F}$ and deglycosylated trastuzumab was exposed to $\mathrm{BCN}$-lissamine (1) in the presence of $\mathrm{mTyr}^{24}$ SDS-PAGE analysis of the process revealed a minor shift for the heavy chain to lower molecular weights after the deglycosylation step, corresponding to the formation of the N297D trastuzumab variant (I $\rightarrow$ II, Figure 2C). Much to our delight, a higher molecular weight band appeared (II $\rightarrow$ III) upon treatment with $\mathrm{mTyr}$ and $\mathbf{1}$. Formation of these antibody derivatives was corroborated as the desired products for each heavy chain by means of fluorescence imaging, HPLC analysis (Figure 2D), and IdeS digestion facilitated LC-MS analysis of the FcFragments (Figure 2E). ${ }^{37}$ No fluorescent band was observed when PNGase F treatment was omitted, thus confirming the importance of tyrosine exposure and the absence of labeling of the untreated antibody. Moreover, we found that labeling of trastuzumab with fluorescent TCO-Alexa Fluor 568 conjugate 2 (TCO-AF568) proceeded in a similar fashion (SI Figure S4). Identical conjugation results were also obtained with cetuximab (SI Figure S6), while experiments with human IgG2 (SI Figure S7, PDB code: 4HAG) and mouse IgG1 (SI
Figure S7, PDB code: 3HKF), both lacking a tyrosine residue neighboring N297, confirmed chemoselectivity for $\mathrm{Tyr}$ residues of the process.

We were intrigued by the fact that only single conjugation of 1 per heavy chain occurred, for both trastuzumab and cetuximab, despite the presence of two $\mathrm{Tyr}$ residues in proximity to the $\mathrm{N}$-glycosylation site. Thus, we undertook the exercise to elucidate whether both Y296 and Y300 were tyrosinase-sensitive by exposing deglycosylated trastuzumab to mTyr in the presence of ascorbic acid as a reducing agent. Under these conditions, a tyrosine $\rightarrow 1,2$-quinone conversion would be immediately followed by 1,2-quinone $\rightarrow$ 1,2-catechol conversion, thereby forming a 3,4-dihydrophenylalanine (DOPA) moiety in the antibody sequence. ${ }^{38}$ Indeed, IdeS digestion followed up by LC-MS analysis of the Fc-fragments revealed the oxidation and reduction of up to two tyrosine residues per chain, resulting in +16 and $+32 \mathrm{Da}$ mass weight increase as determined by LC-MS (SI Figure S8, Table S2). As both Y296 and Y300 appear to be susceptible to tyrosinase oxidation, we assume that after one of the two Tyr residues underwent SPOCQ conjugation to the BCN derivative, access to the other oxidized Tyr residue was mostly blocked and a second modification was prevented. Indeed, we never observed addition of two BCN-lissamine $\mathbf{1}$ derivatives to deglycosylated IgG1, even upon treatment with $>5$ equiv of 1 per Tyr (SI Figure S5). Finally, we applied conjugation conditions optimized in earlier work $\left(\mathrm{pH} 5.5,4{ }^{\circ} \mathrm{C}\right),{ }^{24,25}$ resulting in $95 \%$ conversion with only 2.5 equiv of $\mathrm{BCN}$-lissamine per reactive Tyr residue ( 5 equiv per antibody), and completion of the reaction overnight.

After these promising results, we were keen to explore the suitability of the PNGase F-enabled tyrosinase-mediated click reaction for direct conjugation of cytotoxic linker-drugs to an antibody $\mathrm{Fc}$-domain as a novel approach to conveniently generate functionalized ADCs. To this end, a range of linker- 
Scheme 1. Synthetic Routes to BCN-PBD 5, Linear BCN-MMAE 6 (for DAR2 ADC), and Branched BCN-MMAE 7 (for DAR4 $\mathrm{ADC})^{a}$
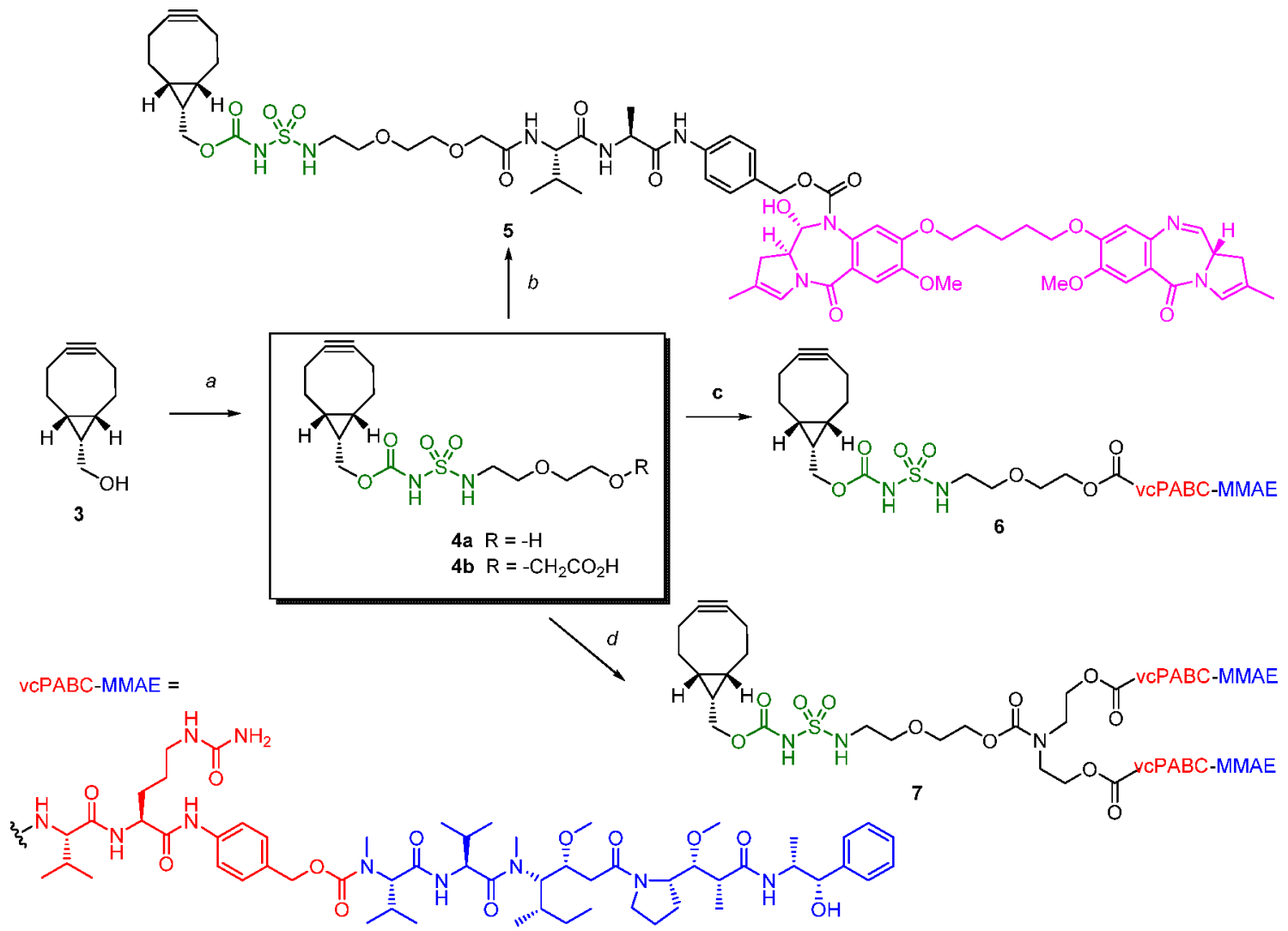

${ }^{a}$ Conditions: (a) chlorosulfonyl isocyanate, $\mathrm{Et}_{3} \mathrm{~N}$, and either then 2-(2-aminoethoxy)ethanol (4a) or 2-(2-(2-aminoethoxy)ethoxy)acetic acid (4b), $\mathrm{MeCN}, 57 \%$ (4a) or 39\% (4b); (b) 4b, H-vaPABC-PBD-amine, EDC.HCl, $\mathrm{CHCl}_{3}, 67 \%$; (c) 4a, p-nitrophenyl chloroformate, $\mathrm{Et}_{3} \mathrm{~N}, \mathrm{DCM}$; then $\mathrm{H}$-vaPABC-MMAE, $\mathrm{Et}_{3} \mathrm{~N}$, DMF, $53 \%$ over 2 steps; (d) 4a, $p$-nitrophenyl chloroformate, $\mathrm{Et}_{3} \mathrm{~N}$, DCM; then diethanolamine, $\mathrm{Et}_{3} \mathrm{~N}$, DMF; then 4 nitrophenyl chloroformate, $\mathrm{Et}_{3} \mathrm{~N}, \mathrm{DCM}$; then $\mathrm{H}$-vaPABC-MMAE, $\mathrm{Et}_{3} \mathrm{~N}, \mathrm{DMF}, 3 \%$ over 4 steps.

payload constructs was synthesized (Scheme 1) by covalent attachment of cytotoxic payloads PBD dimer (5) or MMAE (6 and 7) to BCN derivatives 4a via a Val-Cit-PABC cleavable linker (shown in red) or to $\mathbf{4 b}$ via Val-Ala-PABC. Notably, in each case we also inserted a carbamoyl-sulfamide unit (shown in green), a highly polar linker from medicinal chemistry programs and marketed as HydraSpace technology, ${ }^{39}$ in order to impart better solubility to the $\mathrm{BCN}$-linker-drugs and to improve the putative therapeutic window. In addition, for MMAE we prepared both a linear linker-drug bearing one payload (6) as well as a linker-drug with a branching unit and two payloads (7), in order to generate the respective ADCs with drug-to-antibody ratio (DAR) of 2 or 4, respectively. Next, each linker-drug 5-7 was individually conjugated to deglycosylated trastuzumab in the presence of mushroom tyrosinase, thereby providing the desired ADCs in each case with $>90 \%$ efficiency, resulting in the clean formation of DAR2 or DAR4 ADCs as illustrated by MS, HIC, and HPLC analysis (SI Figures S9-S14). Gratifyingly, the resulting ADCs each showed highly potent, subnanomolar in vitro killing of the HER2-expressing cell line Sk-Br-3 (Figure 3A). Target-specific killing was confirmed by the lack of efficacy of a nonbinding isotype control DAR4MMAE ADC based on B12 (antigp120). Finally, we corroborated that the resulting ADCs completely lack binding capacity to Fc- $\gamma$ RIII, the immune cell receptor responsible for antibody effector functions such as antibody-dependent cellular cytotoxicity (ADCC) and antibody-dependent cellular phagocytosis (ADCP) (Figure 3B).

\section{CONCLUSION}

In conclusion, we have demonstrated that the two-stage sequence of antibody deglycosylation followed by tyrosinasemediated strain-promoted cycloaddition is a convenient way to generate homogeneous and stable antibody-drug conjugates from human IgG1 antibodies without the need for genetic modification. The method can be employed for the direct labeling of antibodies with a range of small molecule probes, including cytotoxic payloads, thereby selectively generating DAR2 or DAR4 ADCs. The resulting ADCs showed potent and target-specific in vitro killing, which warrants further exploration in the field of targeted chemotherapy. We note that the technology described herein for ADCs stands out with regard to the analogous TGase-mediated installation of cytotoxic payload after antibody deglycosylation, ${ }^{36}$ in terms of both the larger number of steps of the latter as well as the fact that a glutamine residing in the antibody binding domain (HC-Q3) may be concomitantly modified by TGase. ${ }^{40}$ Furthermore, the complete nihilation of binding to Fc- $\gamma$ RIIIa is an indication of an anticipated lack of effector function of the $\mathrm{ADCs}$ presented herein, which is often desirable to mitigate Fc- $\gamma$ receptor-mediated toxicities. ${ }^{31}$ Thus, based on the straightforward application to human IgG1 antibodies with many commercially available BCN and TCO-based reagents 
A

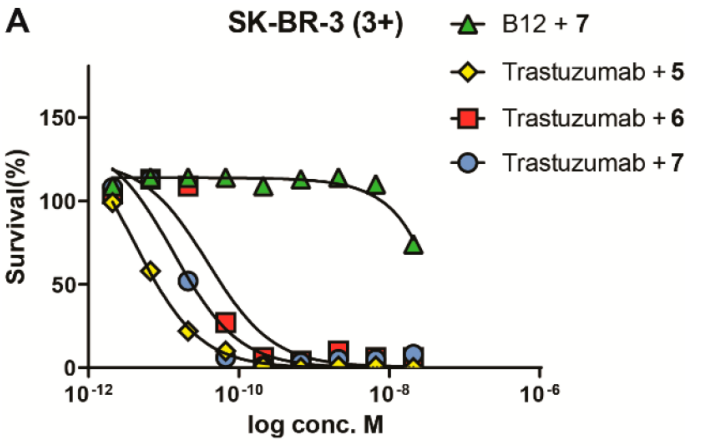

B

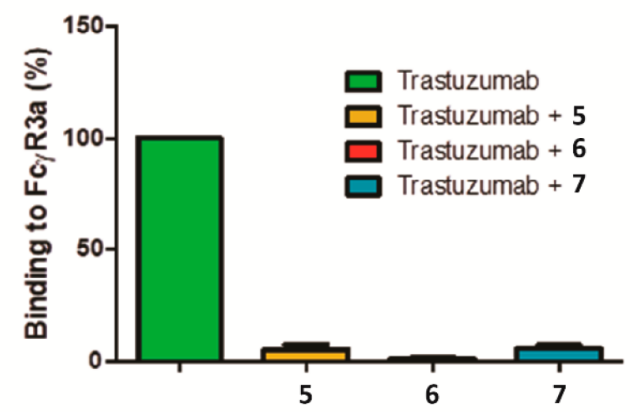

Figure 3. (A) Cell-killing in vitro assay on SK-BR-3. (B) Binding of trastuzumab and $\mathrm{ADCs}$ to $\mathrm{Fc}-\gamma \mathrm{R}$ IIIa. Data is normalized against trastuzumab.

and materials, we anticipate that the technology presented here will find useful application in the fields of antibody-based diagnostics and most promisingly targeted anticancer therapeutics with ADCs. Further applications along these lines are currently being explored in our laboratories.

\section{ASSOCIATED CONTENT}

\section{(1) Supporting Information}

The Supporting Information is available free of charge at https://pubs.acs.org/doi/10.1021/acs.bioconjchem.1c00351.

Additional information on the synthesis of the linker drug conjugates, enzymatic glycan modifications, conjugation experiments, and in vitro assays (PDF)

\section{AUTHOR INFORMATION}

\section{Corresponding Author}

Bauke Albada - Laboratory of Organic Chemistry,

Wageningen University \& Research, 6708 WE Wageningen, The Netherlands; 10 orcid.org/0000-0003-3659-2434;

Email: bauke.albada@wur.nl

\section{Authors}

Jorick J. Bruins - Laboratory of Organic Chemistry, Wageningen University \& Research, 6708 WE Wageningen, The Netherlands

Johannes A. M. Damen - Laboratory of Organic Chemistry, Wageningen University \& Research, 6708 WE Wageningen, The Netherlands

Marloes A. Wijdeven - Synaffix BV, 5349 AB Oss, The Netherlands

Lianne P. W. M. Lelieveldt - Synaffix BV, 5349 AB Oss, The Netherlands

Floris L. van Delft - Laboratory of Organic Chemistry, Wageningen University \& Research, 6708 WE Wageningen,
The Netherlands; Synaffix BV, 5349 AB Oss, The

Netherlands

Complete contact information is available at:

https://pubs.acs.org/10.1021/acs.bioconjchem.1c00351

\section{Notes}

The authors declare the following competing financial interest(s): JB, MW, FvD, and $\mathrm{BA}$ hold a patent on the current invention. FvD is CSO and shareholder of SynAffix BV.

\section{ACKNOWLEDGMENTS}

We kindly acknowledge Inge C. J. Hurkmans for performing the in vitro potency assay. We thank the Institute of Chemical Immunology for their financial support.

\section{REFERENCES}

(1) Tran Cao, H. S.; Kaushal, S.; Metildi, C. A.; Menen, R. S.; Lee, C.; Snyder, C. S.; Messer, K.; Pu, M.; Luiken, G. A.; Talamini, M. A.; et al. Tumor-specific fluorescence antibody imaging enables accurate staging laparoscopy in an orthotopic model of pancreatic cancer. Hepato-Gastroenterology 2011, 59 (118), 1994-1999.

(2) Luo, S.; Zhang, E.; Su, Y.; Cheng, T.; Shi, C. A review of NIR dyes in cancer targeting and imaging. Biomaterials 2011, 32 (29), $7127-7138$.

(3) Wu, A. M.; Olafsen, T. Antibodies for molecular imaging of cancer. Cancer J. 2008, 14 (3), 191-197.

(4) Mishiro, K.; Hanaoka, H.; Yamaguchi, A.; Ogawa, K. Radiotheranostics with radiolanthanides: Design, development strategies, and medical applications. Coord. Chem. Rev. 2019, 383, 104-131.

(5) Chari, R. V. J.; Miller, M. L.; Widdison, W. C. antibody-drug conjugates: an emerging concept in cancer therapy. Angew. Chem., Int. Ed. 2014, 53 (15), 3796-3827.

(6) Cohen, L.; Walt, D. R. Evaluation of Antibody Biotinylation Approaches for Enhanced Sensitivity of Single Molecule Array (Simoa) Immunoassays. Bioconjugate Chem. 2018, 29 (10), 34523458.

(7) Vira, S.; Mekhedov, E.; Humphrey, G.; Blank, P. S. Fluorescentlabeled antibodies: Balancing functionality and degree of labeling. Anal. Biochem. 2010, 402 (2), 146-150.

(8) Spicer, C. D.; Pashuck, E. T.; Stevens, M. M. Achieving Controlled Biomolecule-Biomaterial Conjugation. Chem. Rev. 2018, 118 (16), 7702-7743.

(9) Kohmer, N.; Westhaus, S.; Ruhl, C.; Ciesek, S.; Rabenau, H. F. Clinical performance of different SARS-CoV-2 IgG antibody tests. J. Med. Virol. 2020, 92 (10), 2243-2247.

(10) Metildi, C. A.; Kaushal, S.; Luiken, G. A.; Talamini, M. A.; Hoffman, R. M.; Bouvet, M. Fluorescently labeled chimeric anti-CEA antibody improves detection and resection of human colon cancer in a patient-derived orthotopic xenograft (PDOX) nude mouse model. J. Surg. Oncol. 2014, 109 (5), 451-458.

(11) Mathe, G.; Lou, T. B.; Bernard, J. Effet Sur La Leucemie 1210 De La Souris Dune Combinaison Par Diazotation Da-Methopterine Et De Gamma-Globulines De Hamsters Porteurs De Cette Leucemie Par Heterogreffe. Comptes rendus hebdomadaires des seances de l'Academie des sciences 1958, 246 (10), 1626-1628.

(12) Larson, R. A.; Sievers, E. L.; Stadtmauer, E. A.; Lowenberg, B.; Estey, E. H.; Dombret, H.; Theobald, M.; Voliotis, D.; Bennett, J. M.; Richie, M.; et al. Final report of the efficacy and safety of gemtuzumab ozogamicin (Mylotarg) in patients with CD33-positive acute myeloid leukemia in first recurrence. Cancer 2005, 104 (7), 1442-1452.

(13) Younes, A.; Bartlett, N. L.; Leonard, J. P.; Kennedy, D. A.; Lynch, C. M.; Sievers, E. L.; Forero-Torres, A. Brentuximab Vedotin (SGN-35) for Relapsed CD30-Positive Lymphomas. N. Engl. J. Med. 2010, 363 (19), 1812-1821. 
(14) Joubert, N.; Beck, A.; Dumontet, C.; Denevault-Sabourin, C. Antibody-Drug Conjugates: The Last Decade. Pharmaceuticals 2020, 13 (9), 245

(15) Zammarchi, F.; Corbett, S.; Adams, L.; Tyrer, P. C.; Kiakos, K.; Janghra, N.; Marafioti, T.; Britten, C. E.; Havenith, C. E. G.; Chivers, $S$; et al. ADCT-402, a PBD dimer-containing antibody drug conjugate targeting CD19-expressing malignancies. Blood 2018, 131 (10), 1094-1105.

(16) Junutula, J. R.; Raab, H.; Clark, S.; Bhakta, S.; Leipold, D. D.; Weir, S.; Chen, Y.; Simpson, M.; Tsai, S. P.; Dennis, M. S.; et al. Sitespecific conjugation of a cytotoxic drug to an antibody improves the therapeutic index. Nat. Biotechnol. 2008, 26 (8), 925-932.

(17) Pillow, T. H.; Tien, J.; Parsons-Reponte, K. L.; Bhakta, S.; Li, H.; Staben, L. R.; Li, G.; Chuh, J.; Fourie-O’Donohue, A.; Darwish, M.; et al. Site-specific trastuzumab maytansinoid antibody-drug conjugates with improved therapeutic activity through linker and antibody engineering. J. Med. Chem. 2014, 57 (19), 7890-7899.

(18) Chin, J. W.; Santoro, S. W.; Martin, A. B.; King, D. S.; Wang, L.; Schultz, P. G. Addition of p-azido-L-phenylalanine to the genetic code of Escherichia coli. J. Am. Chem. Soc. 2002, 124 (31), 90269027

(19) Hallam, T. J.; Wold, E.; Wahl, A.; Smider, V. V. Antibody Conjugates with Unnatural Amino Acids. Mol. Pharmaceutics 2015, 12 (6), 1848-1862.

(20) van Berkel, S. S.; van Delft, F. L. Enzymatic strategies for (near) clinical development of antibody-drug conjugates. Drug Discovery Today: Technol. 2018, 30, 3-10.

(21) Zhang, Y.; Park, K. Y.; Suazo, K. F.; Distefano, M. D. Recent progress in enzymatic protein labelling techniques and their applications. Chem. Soc. Rev. 2018, 47 (24), 9106-9136.

(22) Boutureira, O.; Bernardes, G. J. L. Advances in Chemical Protein Modification. Chem. Rev. 2015, 115 (5), 2174-2195.

(23) Oliveira, B. L.; Guo, Z.; Bernardes, G. J. L. Inverse electron demand Diels-Alder reactions in chemical biology. Chem. Soc. Rev. 2017, 46 (16), 4895-4950.

(24) Bruins, J. J.; Westphal, A. H.; Albada, B.; Wagner, K.; Bartels, L.; Spits, H.; van Berkel, W. J. H.; van Delft, F. L. Inducible, SiteSpecific Protein Labeling by Tyrosine Oxidation-Strain-Promoted (4 + 2) Cycloaddition. Bioconjugate Chem. 2017, 28 (4), 1189-1193.

(25) Bruins, J. J.; Blanco-Ania, D.; van der Doef, V.; van Delft, F. L.; Albada, B. Orthogonal, dual protein labelling by tandem cycloaddition of strained alkenes and alkynes to ortho-quinones and azides. Chem. Commun. 2018, 54 (53), 7338-7341.

(26) Borrmann, A.; Fatunsin, O.; Dommerholt, J.; Jonker, A. M.; Lowik, D. W. P. M.; van Hest, J. C. M.; van Delft, F. L. Strainpromoted oxidation-controlled cyclooctyne-1,2-quinone cycloaddition (SPOCQ) for fast and activatable protein conjugation. Bioconjugate Chem. 2015, 26 (2), 257-261.

(27) Bruins, J. J.; Albada, B.; van Delft, F. ortho-Quinones and Analogues Thereof: Highly Reactive Intermediates for Fast and Selective Biofunctionalization. Chem. - Eur. J. 2018, 24 (19), 47494756.

(28) Albada, B.; Keijzer, J. F.; Zuilhof, H.; van Delft, F. OxidationInduced "One-Pot" Click Chemistry. Chem. Rev. 2021, 121 (12), 7032-7058.

(29) Buecheler, J. W.; Winzer, M.; Tonillo, J.; Weber, C.; Gieseler, H. Impact of Payload Hydrophobicity on the Stability of antibodydrug Conjugates. Mol. Pharmaceutics 2018, 15 (7), 2656-2664.

(30) Su, D.; Kozak, K. R.; Sadowsky, J.; Yu, S. F.; FourieO’Donohue, A.; Nelson, C.; Vandlen, R.; Ohri, R.; Liu, L.; Ng, C.; et al. Modulating antibody-drug Conjugate Payload Metabolism by Conjugation Site and Linker Modification. Bioconjugate Chem. 2018, 29 (4), 1155-1167.

(31) Strop, P.; Liu, S. H.; Dorywalska, M.; Delaria, K.; Dushin, R. G.; Tran, T. T.; Ho, W. H.; Farias, S.; Casas, M. G.; Abdiche, Y.; et al. Location Matters: Site of Conjugation Modulates Stability and Pharmacokinetics of Antibody Drug Conjugates. Chem. Biol. (Oxford, U. K.) 2013, 20 (2), 161-167.
(32) Subedi, G. P.; Barb, A. W. The Structural Role of Antibody NGlycosylation in Receptor Interactions. Structure 2015, 23 (9), 15731583.

(33) Tumey, L. N.; Leverett, C. A.; Vetelino, B.; Li, F. P.; Rago, B.; Han, X. G.; Loganzo, F.; Musto, S.; Bai, G. Y.; Sukuru, S. C. K.; et al. Optimization of Tubulysin antibody-drug Conjugates: A Case Study in Addressing ADC Metabolism. ACS Med. Chem. Lett. 2016, 7 (11), 977-982.

(34) Zhou, Q.; Stefano, J. E.; Manning, C.; Kyazike, J.; Chen, B.; Gianolio, D. A.; Park, A.; Busch, M.; Bird, J.; Zheng, X.; et al. Sitespecific antibody-drug conjugation through glycoengineering. Bioconjugate Chem. 2014, 25 (3), 510-520.

(35) van Geel, R.; Wijdeven, M. A.; Heesbeen, R.; Verkade, J. M. M.; Wasiel, A. A.; van Berkel, S. S.; van Delft, F. L. Chemoenzymatic Conjugation of Toxic Payloads to the Globally Conserved N-Glycan of Native mAbs Provides Homogeneous and Highly Efficacious antibody-drug Conjugates. Bioconjugate Chem. 2015, 26 (11), 22332242.

(36) Jeger, S.; Zimmermann, K.; Blanc, A.; Grunberg, J.; Honer, M.; Hunziker, P.; Struthers, H.; Schibli, R. Site-Specific and Stoichiometric Modification of Antibodies by Bacterial Transglutaminase. Angew. Chem., Int. Ed. 2010, 49 (51), 9995-9997.

(37) Bruins, J. J.; van de Wouw, C.; Keijzer, J. F.; Albada, B.; van Delft, F. L. Inducible, Selective Labeling of Proteins via Enzymatic Oxidation of Tyrosine. In Enzyme-Mediated Ligation Methods, Nuijens, T.; Schmidt, M., Eds.; Springer New York: New York, NY, 2019; pp 357-368.

(38) Struck, A. W.; Bennett, M. R.; Shepherd, S. A.; Law, B. J. C.; Zhuo, Y.; Wong, L. S.; Micklefield, J. An Enzyme Cascade for Selective Modification of Tyrosine Residues in Structurally Diverse Peptides and Proteins. J. Am. Chem. Soc. 2016, 138 (9), 3038-3045.

(39) Verkade, J. M. M.; Wijdeven, M. A.; van Geel, R.; Janssen, B. M. G.; van Berkel, S. S.; van Delft, F. L. A Polar Sulfamide Spacer Significantly Enhances the Manufacturability, Stability, and Therapeutic Index of Antibody-Drug Conjugates. Antibodies 2018, 7 (1), 12.

(40) Marculescu, C.; Lakshminarayanan, A.; Gault, J.; Knight, J. C.; Folkes, L. K.; Spink, T.; Robinson, C. V.; Vallis, K.; Davis, B. G.; Cornelissen, B. Probing the limits of Q-tag bioconjugation of antibodies. Chem. Commun. 2019, 55 (76), 11342-11345. 